\title{
Study of enamel and dentin in primary teeth with dentinogenesis imperfecta type I: A case report
}

\author{
Yoko Kamasaki*, Keigo Imamura, Haruka Nishimata, Miyuki Nishiguchi and Taku Fujiwara \\ Department of Paediatric Dentistry, Nagasaki University Graduate School of Biomedical Science, Nagasaki, Japan
}

\begin{abstract}
We conducted morphologic investigation of dentinogenesis imperfecta (DI) primary teeth of a 10-year-old patient with osteogenesis imperfecta (OI) using light microscopy and scanning electron microscopy and then performed mineral composition analysis of dentin using Raman spectroscopy. In the DI teeth, a number of cracks and fractures were observed not only in the dentin but in the enamel, and irregular dentinal tubules were remarkable in the internal area of the mantle dentin layer. The circumpulpal dentin of DI teeth was significantly different from that of the control teeth. Results of Raman analysis showed that quantitative differences in the mineral content (carbonate and phosphate) between DI teeth and the control were not significant. However, the amount of matrix (amide I of collagen) was decreased in DI teeth. The DI teeth were found to have increased fragility in both dentin and enamel, it might be due to decreased type I collagen formation.
\end{abstract}

\section{Introduction}

Dentinogenesis imperfecta (DI) is a rare genetic disease affecting the development of dentin, and it is clinically characterized by tooth discoloration and early enamel loss. Many studies about DI have been conducted over the last few decades [1-9]. DI-type I may be seen in conjunction with osteogenesis imperfecta (OI), which is a disease characterized by fragile bones resulting from genetic mutations that impair collagen synthesis [2,7]. DI-type I teeth are found to have abnormalities in the dentinal matrix and tubules with normal layers of mantle dentin $[2,5,6]$.

Clinically, DI teeth are associated with esthetic problems resulting from tooth discoloration, especially in the primary dentition. Functional problems include enamel loss due to significant attrition, which often leads to exposure of dentin, followed by exposure of pulp, and a reduced occlusal vertical dimension. In severe cases at risk of dental wear, preventive intervention using full coverage restoration is recommended before problems occur [1].

Given that DI-type I teeth, are characterized by the presence of abnormal dentin, it is unclear why early significant attrition occurs. This finding suggests there are potential abnormalities involving the enamel as well. However, to the best of our knowledge, there are no reports demonstrating clear histological abnormalities of the enamel in patients with this disease.

Many studies have examined the exfoliated primary teeth from patients with DI $[2,5,8,9]$. However, in those studies, the enamel of severe cases had already been lost and could not be evaluated. On the other hands, the enamel of mild cases may have exhibited minimal changes, suggesting that no abnormalities of the enamel could be found. Thus, it is worth investigating the exfoliated primary teeth of patients with DI that appear discoloured with no evidence of significant enamel loss. We had the opportunity to extract primary teeth from a girl diagnosed with OI, which were found to be discoloured with little enamel loss. We conducted morphologic investigation of the DI teeth using light microscopy and scanning electron microscopy and then performed mineral composition analysis of dentin using Raman spectroscopy.

\section{Case report of DI teeth associated with OI}

A 10-year-old girl with OI presented to our clinic (Nagasaki, Japan) with crowding of primary teeth. More specifically, her mandibular right primary canine and first molar were crowded out of the dentition by an erupting succedaneous canine and first premolar. On examination, these teeth, including the other remaining primary teeth, were yellowbrown in colour and exhibited an amber translucency, which led us to suspected they were affected by DI associated with OI. The erupted permanent incisors also exhibited a greyish colour, though the change of colour was milder than that of the primary teeth. Both the mandibular right primary canine and the primary first molar were extracted, and then examined morphologically and compositionally with consent of the patient and her mother. We compared the morphological and mineral compositional findings between the DI primary teeth extracted from this patient and the respective teeth obtained from a healthy 10-year-old girl.

\section{Observation of tooth surface}

The mandibular right primary canine and first molar from a 10-year-old girl with OI and the respective teeth from a healthy 10-year-old girl were observed using a stereomicroscope and compared (Figure 1). Examination of the DI teeth revealed smooth surface with

*Correspondence to: Yoko Kamasaki, Department of Paediatric Dentistry, Nagasaki University Graduate School of Biomedical Science, 1-7-1 Sakamoto, Nagasaki 852-8588, Japan, Tel: +81-95-819-7675; Fax: +81-95-819-7675; E-mail: ichiro@nagasaki-u.ac.jp

Key words: dentinogenesis imperfecta, osteogenesis imperfecta, dentin, enamel, raman analysis

Received: January 19, 2019; Accepted: January 28, 2019; Published: January 31,2019 
yellow-brown discoloration on the labial surface, a rough and coarse surface on the cervical crown, and many vertical enamel cracks.

\section{Observation of undecalcified section}

The teeth were embedded in methyl methacrylate (MMA) and cut longitudinally in a mesiodistal direction. The 25-35 $\mu \mathrm{m}$ thick slices of sections were prepared from each block embedded tooth using a special cutting and grinding machine (EXAKT GmbH, Germany) and stained with hematoxylin and eosin. The sections were examined using light microscopy (Axiovert 200, Carl Zeiss, Jena, Germany) (Figure 2). The enamel thickness of the DI teeth was comparable to that of the control teeth; small loss of enamel due to attrition was observed in both the control and DI teeth. In the enamel surface of DI teeth, deepstained layers were observed in the cervical crown area (Figure 2-h). Dentin-enamel junction (DEJ) in the DI primary canine separated in some areas, but not all. However, in DI teeth, cracks were observed in the enamel adjacent to the DEJ and enamel surface (Figure 2-d,h arrowheads). Furthermore, in the DI teeth, many cracks ware observed within the dentin covered with enamel. The course of dentinal tubules of DI teeth appeared periodically disorganized and were observed as dark lines that were serial with adjacent tubules. In DI teeth, the circumpulpal dentin in both DI teeth was also very irregular. Secondary dentin was observed in the control teeth; however, none was observed in the DI teeth.

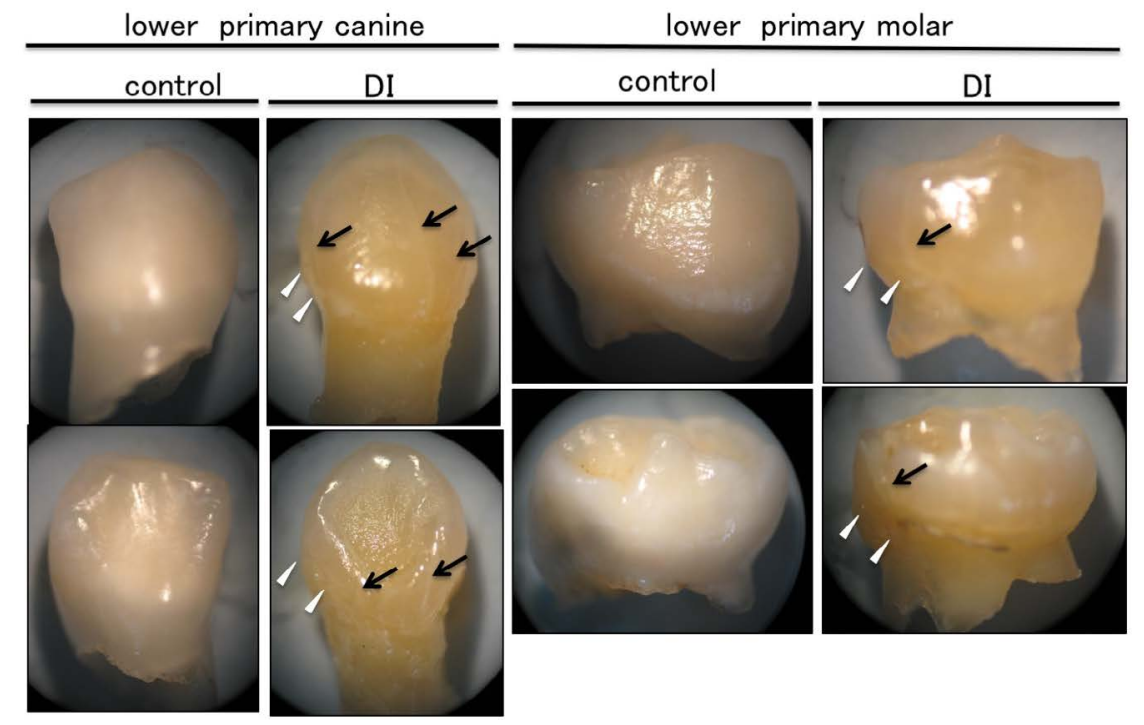

Figure 1. Photographs from the stereomicroscope. Arrows indicate an enamel crack. Arrowheads indicate rough surface areas.
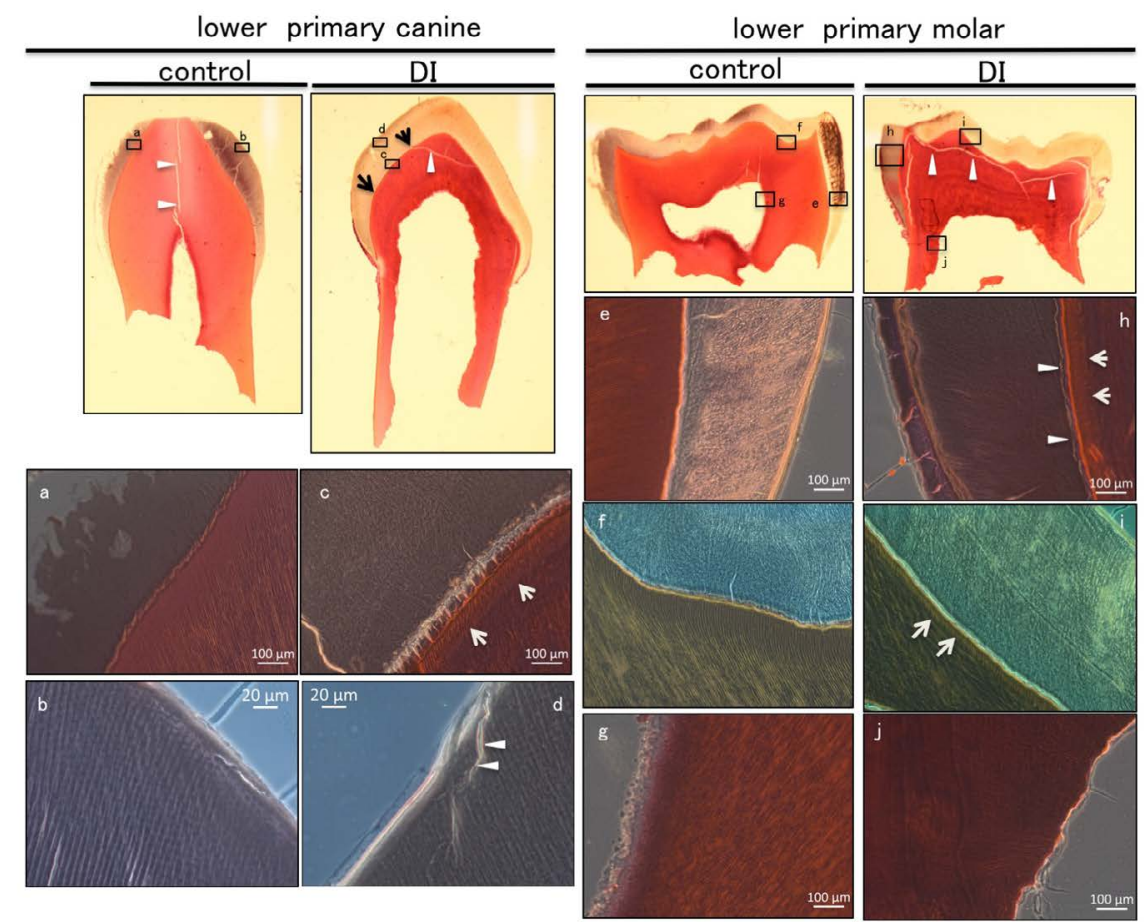

Figure 2. Photographs from the light microscope. Black arrows indicate DEJ areas that appear separated. Arrowheads indicate cracks in the dentin. The higher magnification images of the boxes in each photo are showed in a-j. 


\section{Dentin mineral composition analysis using Raman spectroscopy}

The remaining tooth samples embedded in MMA were finely polished using carbide papers with increasing grit sizes (from 600 up to 4000 grit) and then analyzed for dentin mineral composition using a DXR Raman microscope (Thermo Scientific, Waltham, MA, USA). A 780-nm wavelength laser was used as an exciting source and the resulting average spot diameter measured $1.6 \mu \mathrm{m}$. The Raman spectra of five points from each specimen-located in the middle of the coronal dentin-were analyzed using the OMNIC software program (Thermo Scientific, Waltham, MA, USA). The relative peak intensities of each band from the Raman spectrum were compared between the DI dentin and the control dentin. The major components of dentin, including mineral phosphate, carbonate, and matrix collagen, were observed spectroscopically. Dividing the primary phosphate band (approximately $959 \mathrm{~cm}^{-1}$ ) by the amide I band $\left(1616-1720 \mathrm{~cm}^{-1}\right.$ ) corresponds to the mineral-to-matrix ratio, which indicates the amount of mineralization [10]. The carbonate band (approximately $1070 \mathrm{~cm}^{-1}$ ) shows that phosphate positions in the apatitic lattice are susceptible to ionic substitutions [10]. The width of the primary phosphate band near $959 \mathrm{~cm}^{-1}$ indicates mineral crystallinity [10].

Dentin of DI teeth, both in the primary canine and first premolar, showed a significantly higher value in mineral-to-matrix ratio than that of the control teeth (Figure 3-A). However, there was no significant difference observed in the carbonate-to-phosphate ratio and the mineral crystallinity between the DI and control teeth (Figure 3-B,C).

\section{Observation by SEM}

After Raman analysis, each specimen was coated with gold and the microstructure of enamel and dentin was examined using scanning electron microscopy (SEM) (S-3500, Hitachi Ltd., Tokyo, Japan) (Figure 4). To observe the enamel and dentin clearly, the specimens were treated with $40 \%$ phosphoric acid for $10 \mathrm{~s}$ prior to coating with gold.

SEM imaging revealed that the dentin microstructure differed markedly between the DI and control teeth. In the DI dentin, the tubule distribution pattern appeared less ordered when compared with the control dentin (Figure 4-C). The number of cracks identified in the DI dentin was greater than that of the control dentin. Cracks in the enamel were observed only in DI teeth, and most cracks occurred in the enamel rather than at the DEJ (Figure 4-D,E,F).

\section{Discussion}

Variations in the clinical features of DI teeth have been reported. It is well known that the primary teeth are more severely affected than the permanent teeth in the same individual $[1,3,6,7]$. The correlation between the severity of DI manifestations and the severity of OI symptoms has been investigated, but not determined [4]. Characteristic discoloration in DI is reported as opalescent, grey, brown, or yellow [7]. Although these discolorations were reported to be the result of light diffraction through the defective DEJ, the true reason for tooth discoloration or the shearing of enamel is still unclear [2]. It was reported that an increased incidence of severe attrition and enamel fracture was observed almost exclusively in the primary teeth with yellowbrown discoloration but not with opalescent grey discoloration [7].

In the present case, we could investigate the primary DI teeth with yellow-brown discoloration without significant enamel loss and some weak areas were observed in those. A number of cracks in the
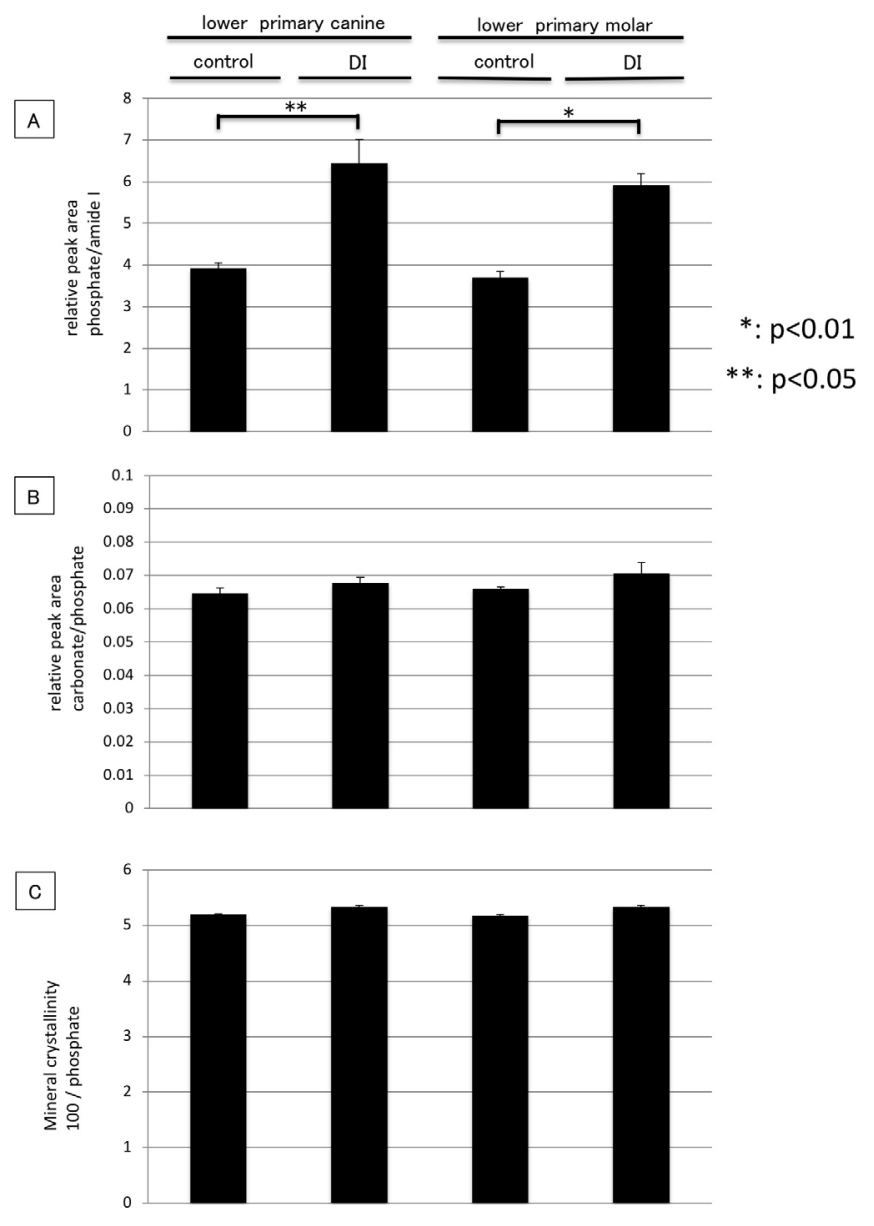

Figure 3. Raman spectroscopy analysis of dentin

Bar graphs representing (A) relative peak areas of bands of phosphate and amide I, (B) relative peak areas of bands of carbonate and phosphate, $(\mathrm{C})$ inverse full width at halfmaximum of bands of phosphate.
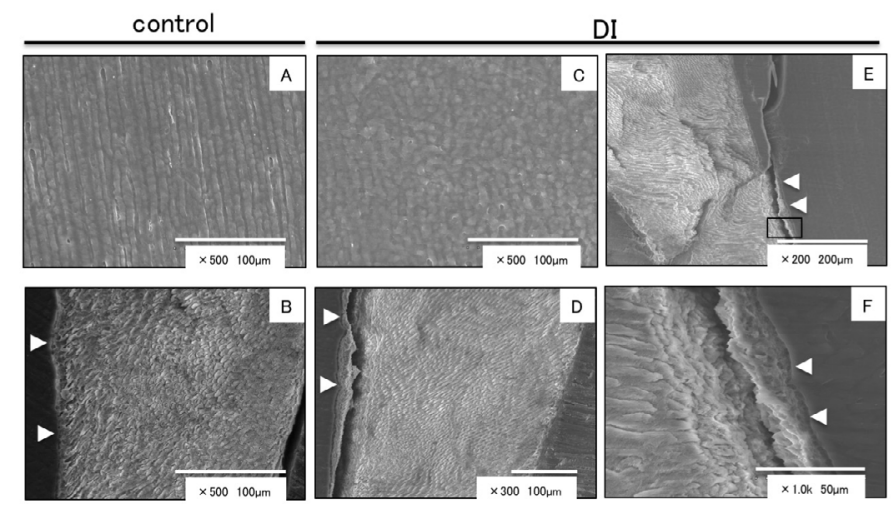

Figure 4. Photographs from SEM. Dentin (A) and enamel (B) of the control tooth (primary canine). C, D, E, and F are those of a DI canine. F is a higher magnification of the box in $\mathrm{E}$. Arrowheads indicate the D,E,J

enamel surface were observed by stereomicroscope. Observation with light microscopy revealed that parts of the enamel structure in DI teeth resembled the structure in the control teeth, in which enamel rods with cross striation were arranged from the DEJ to the enamel surface. However, in DI teeth, cracks were observed in the enamel adjacent to the DEJ and enamel surface. The enamel of DI teeth was found to be 
more brittle when compared to the control teeth. In addition to color, the enamel surface quality of the DI teeth was different from the control teeth; DI teeth had both smooth and rough surfaces, the cervical area of the DI teeth was found to be very rough. Observation with light microscopy also revealed the outer layer of the enamel surface in the cervical area of DI teeth differed markedly in structure and stained deeply, indicating low mineralization. Recent studies have reported that, during the maturation stage, ameloblasts remain active secretory cells and secrete some proteolytic enzymes whose function is to remove organic matrix [11]. Dysfunction of ameloblasts during the maturation stage may cause the formation of the abnormal enamel surface.

Observation with light microscopy revealed a wide range of visible cracks in the dentin covered with enamel, which appeared to be a characteristic finding in DI teeth. It was evident that the dentin of DI teeth was brittle when compared to control teeth. However, the results of the Raman analysis performed in this study showed that the mineral-to-matrix ratio of DI teeth was higher than the control teeth, which indicated a higher degree of mineralization in DI teeth. Given that the quantitative and qualitative difference of mineral (carbonate and phosphate) between DI teeth and the control was found to be negligible, it is presumed that the high mineral-to-matrix ratio resulted from a low amount of matrix (amide I of collagen) in DI teeth. The present case is associated with OI, and mild cases of this disease are usually caused by a defect in COL1A1 [12], which reduces the amount of normal type I collagen formation. It was suggested that a low amount of matrix in DI teeth arose from reduced type I collagen formation and affected on fracture resistance of DI dentin.

It has been reported that the enamel is normal but tends to fracture away, and that early enamel loss results from rupture in the DEJ [13]. There are other reports showing that the enamel of DI-type I teeth is normal [2,5]; however, in the present case, enamel fractures were shown to occur mainly along major incremental lines within the enamel and partly at the DEJ. Those findings suggested that the enamel of DI teeth might be lost easily due to the fragility of enamel, which then exposed dentin more easily. It was suggested that a defect in COL1A1 affected not only dentin, but also enamel during tooth germ development. However, this report has a limitation, we used DI teeth from only one patient, and our results must not be representative of all DI-type I teeth. Enamel malformations associated with DI-type II, which is caused by dentin sialophosphoprotein (DSPP) mutation have also been reported [14]. Thus, further study with many more subjects is needed for better understanding of DI-type I teeth.

\section{References}

1. American Academy of Pediatric Dentistry (2016) Guideline on Dental Management of Heritable Dental Developmental Anomalies. Pediatr Dent 38: 302-307. [Crossref]

2. Lindau BM, Dietz W, Hoyer I, Lundgren T, Storhaug K, et al. (1999) Morphology of dental enamel and dentine-enamel junction in osteogenesis imperfecta. Int $J$ Paediatr Dent 9: 13-21. [Crossref]

3. Luder HU, van Waes H, Raghunath M, Steinmann B (1996) Mild dental findings associated with severe osteogenesis imperfecta due to a point mutation in the alpha 2(I) collagen gene demonstrate different expression of the genetic defect in bone and teeth. J Craniofac Genet Dev Biol 16: 156-163. [Crossref]

4. Lund AM, Jensen BL, Nielsen LA, Skovby F (1998) Dental manifestations of osteogenesis imperfecta and abnormalities of collagen I metabolism. J Craniofac Genet Dev Biol 18: 30-37. [Crossref]

5. Lygidakis NA, Smith R, Oulis CJ (1996) Scanning electron microscopy of teeth in osteogenesis imperfecta type I. Oral Surg Oral Med Oral Pathol Oral Radiol Endod 81: 567-572. [Crossref]

6. Waltimo J, Ojanotko-Harri A, Lukinmaa PL (1996) Mild forms of dentinogenesis imperfecta in association with osteogenesis imperfecta as characterized by light and transmission electron microscopy. J Oral Pathol Med 25: 256-264. [Crossref]

7. O'Connell AC, Marini JC (1999) Evaluation of oral problems in an osteogenesis imperfecta population. Oral Surg Oral Med Oral Pathol Oral Radiol Endod 87: 189196. [Crossref]

8. Davis GR, Fearne JM, Sabel N, Norén JG (2015) Microscopic study of dental hard tissues in primary teeth with Dentinogenesis Imperfecta Type II: Correlation of 3D imaging using X-ray microtomography and polarising microscopy. Arch Oral Biol 60: 1013-1020. [Crossref]

9. Min B, Song JS, Lee JH, Choi BJ, Kim KM, et al. (2014) Multiple teeth fractures in dentinogenesis imperfecta: a case report. J Clin Pediatr Dent 38: 362-365. [Crossref]

10. Morris MD, Mandair GS (2011) Raman assessment of bone quality. Clin Orthop Relat Res 469: 2160-2169. [Crossref]

11. Lacruz RS, Nanci A, Kurtz I, Wright JT, Paine ML (2010) Regulation of pH During Amelogenesis. Calcif Tissue Int 86: 91-103. [Crossref]

12. Gajko-Galicka A (2002) Mutations in type I collagen genes resulting in osteogenesis imperfecta in humans. Acta Biochim Pol 49: 433-441. [Crossref]

13. Wright JT, Gantt DG (1985) The ultrastructure of the dental tissues in dentinogenesis imperfecta in man. Arch Oral Biol 30: 201-206. [Crossref]

14. Wang SK, Chan HC, Rajderkar S, Milkovich RN, Uston KA, et al. (2011) Ename malformations associated with a defined dentin sialophosphoprotein mutation in two families. Eur J Oral Sci 119: 158-167. [Crossref]

Copyright: (2019 Kamasaki Y. This is an open-access article distributed under the terms of the Creative Commons Attribution License, which permits unrestricted use, distribution, and reproduction in any medium, provided the original author and source are credited. 